\title{
Community Health Global Network and Sustainable
}

\section{Development}

\section{Rebekah Young ${ }^{a}$, Nicole Hughes ${ }^{b}$, Elizabeth Wainwright ${ }^{c}$, Ted Lankester $^{d}$, Nathan Grills ${ }^{e}$}

${ }^{a}$ MD candidate, The Nossal Institute for Global Health, The University of Melbourne, Australia

${ }^{\mathrm{b}}$ Research assistant at the Nossal Institute for Global Health, The University of Melbourne, Australia

${ }^{c} M S c$, NCTA, Managing Director, Community Health Global Network, London, UK ${ }^{d}$ MA, MB, BChir, MRCGP, RCPSGlasg is founder of Community Health Global Network and Director of Health Services, Interhealth, UK

${ }^{\mathrm{e}}$ MBBS, MPH, DPhil, Associate Editor, CJGH; Nossal Institute of Global Health, University of Melbourne, Australia

\section{Introduction}

With the achievements, failures, and passing of the Millennium Development Goals (MDGs), the world has turned its eyes to the Sustainable Development Goals (SDGs), designed to foster sustainable social, economic, and environmental development over the next 15 years. ${ }^{1}$ Communityled initiatives are increasingly recognised as playing a key role in sustainable community development and in aspirations for universal healthcare. ${ }^{2}$ In many parts of the world, faith-based organisations are some of the main players in community-led development and health care. ${ }^{3}$ Community Health Global Network (CHGN) creates links between organisations, with the purpose being to encourage communities to recognise their assets and abilities, identify shared concerns, and discover solutions together, in order to define and lead their futures in sustainable ways. ${ }^{4} \mathrm{CHGN}$ has facilitated the development of collaborative groups of health and development initiatives called "Clusters" in several countries, including India, Bangladesh, Kenya, Tanzania, Zambia, and Myanmar. In March 2016, these Clusters met together in an International Forum to share learnings, experiences, challenges, achievements, and to encourage one another (Figure 1). Discussions held throughout the forum suggest that the CHGN model is helping to promote effective, sustainable development and health care provision on both a local and a global scale.

\section{Background}

The Millennium Development Goals (MDG) led to significant achievements in addressing extreme poverty across the world, yet persistent inequality and widespread poverty endures. ${ }^{5}$ The world has now turned its eyes to the transition from MDGs to the Sustainable Development Goals (SDGs), designed to foster sustainable social, economic, and environmental development over the next 15 years through investments in education, health, equitable growth, and sustainable production and consumption. ${ }^{1}$ Although health and health care receive little specific attention within the SDGs, the goals illustrate an understanding that the determinants of health are complex and multifactorial, and include interactions between economic, environmental, and social factors, which contribute to the overall health and wellbeing of communities. ${ }^{1,6,7}$ Community-based and community-led approaches, in addition to policy and top-down approaches, will be important in working towards sustainable health and development in the coming years. ${ }^{8}$ In many parts of the world, faith-based groups are some of the most active groups working at the community level to promote health and development. In a develop-

May 2016. Christian Journal for Global Health, 3(1): 89-94. 
ing context, local faith-based organizations can be uniquely placed to contribute to meeting the developmental needs of communities and are increasingly recognised as key contributors to sustaining progress in development. ${ }^{9}$ However, small community organizations, such as these faith-based organizations are often ill-equipped to promote large-scale changes independently. Collaborations between community organisations, and with the formal health systems, are needed if they are to play an active role working towards long term, sustainable development.

\section{Community Health Global Network}

The Community Health Global Network (CHGN) was first conceived in 2005 after observing that many community-based organisations working in similar areas, or delivering similar health-based initiatives, lacked any formal collaboration with one another. CHGN founder, Dr Lankester, commented that these organisations were "often not linked with other charities or local government services and (were) usually unaware that 10 miles down the track another group (were) doing almost exactly the same thing for the community they (were) serving." " CHGN was created out of a desire to link together community organisations and to develop low-cost, high-impact Clusters for the purpose of collaboration, knowledge, and resource sharing around community health. This collaboration facilitates CHGN's ultimate goal of promoting health, wellbeing, and happiness for communities around the world. CHGN recognises that through cooperation and collaboration, Clusters can achieve far more for communities than any individual organisation acting on its own. ${ }^{4}$ To be effective, Clusters should be built on trusting relationships whereby diverse organisations can come together in unity to work toward shared goals and visions.
CHGN supports the formation of Clusters as they work together for the benefit of the local community. In the several years since its initial formation, CHGN has supported the formation of Clusters in several countries around the world, including in India, Bangladesh, Kenya, Zambia, Tanzania, and Myanmar. Aside from helping to establish and support the formation of countrybased Clusters, and thus locally-led health and development, an important role of CHGN is to link together Clusters from different countries to promote sustainable health and development on a global scale. In March 2016, representatives from each of these partner Clusters met together in India for the inaugural international CHGN forum to collaborate and share learning and experiences. The discussions held during this international forum highlighted how Cluster-based community development can contribute to the realisation of the SDGs by promoting greater coordination between grassroots organisations, strengthening the voice of the community on important issues, and increasing integration between the community, government, and other health system players.

\section{What are the CHGN Clusters doing and achieving?}

The CHGN global forum was an opportunity to bring together partners from around the world to learn from each other through the work that is taking place within each individual Cluster. This three-day event held in Dehradun, India was encouraging and reinforced the capacity that Clusters have to contribute to achieving sustainable development. Each Cluster has a different focus, skill set, goals, and method of collaboration. Therefore, each Cluster leads different initiatives for health and development that are specific to their own cultural context. Two case examples of Cluster work are shown below.

May 2016. Christian Journal for Global Health, 3(1): 89-94. 
Case Example 1: The Kenya Cluster

Since its formation in 2014, the Kenya Cluster has worked with its member organisations to coordinate health care delivery. Through the development of sub-Clusters in different locations throughout Kenya, the group has worked to foster relational collaboration despite the fact that member organisations are geographically dispersed. The Cluster has been involved in a variety of different community-based interventions including training community health workers who are able to provide sustainable, low-cost health care. They have also been involved in health promotion and education activities by engaging with community organisations such as schools and training institutions. Through these networks, the Cluster has provided education around reproductive health and HIV. The Cluster has also focused on disability awareness and youth empowerment.

\section{Case Example 2: The Uttarkhand Cluster}

The Uttarakhand Cluster in North India is the oldest and most well-established Cluster involved in CHGN. Formed in 2008, the Uttarakhand Cluster now comprises 50 partner organisations and is involved in a range of different activities including disability inclusion, mental health education, tobacco control, and disaster relief. In 2013, the Cluster mobilised its resources to respond to the North Indian floods, providing relief and assistance to over 600 villages. Because of its reach and reputation, the Uttarakhand Cluster has been contracted by the Indian government to provide services on a local scale.

\section{Approaches to promoting collaborative capital}

Feedback from the participants at the International forum highlighted the importance of common values in promoting cooperation. In some Clusters, it was reported that a common faith background between organisations was a strong platform for relational collaboration within the Cluster. The idea of trust and unity between organisations was identified as being key to developing effective collaboration and repeatedly emerged as playing a key role in making CHGN what it is. CHGN Cluster representatives identified that because of their shared values, organisations within Clusters were able to quickly develop trusting relationships with one another. This trust was built on an understanding that each contributor shared a common vision to work together for the benefit of the other rather than for the benefit of self.

Members identified that their Clusters differed from many other community-led initiatives in terms of their approaches (i.e., a relational and collaborative approach) and achievements (i.e., Clusters reported achieving changes in community health where other organisations had not in the past). They described the importance of sharing ideas with each other to increase capacity in their individual programs. They also described examples where coming together had facilitated their work with the government on programs such as disability and tobacco control, as in the case of the Uttarakhand Cluster. Sharing experiences such as these encouraged the smaller and newer Clusters to consider how they could better integrate with the government health and development activities within their country.

A number of the Clusters shared that they are more effective when each organisation contributes its own strength to the common program. Some Cluster members described their function with reference to 1 Corinthians 12:12 as a body that has many parts but comprises a unified whole. ${ }^{11}$ This imagery encapsulates the belief that each individual part (i.e., each member organisation or individual contributor) may have a different function, but each is valuable and necessary for the body (i.e., the whole Cluster and 
CHGN) to function. Recognising the unique role and worth of each contributor allows organisations to work together rather than competing with one another. Cooperation between Clusters, and within Clusters, was usually very open, with resources and knowledge being freely shared for the benefit of Cluster communities and the CHGN body as a whole.

\section{New ways forward}

CHGN is currently exploring how better to engage with non-faith based organisations, or indeed organisations of diverse faith backgrounds, without compromising the trust and common values that have been integral to a number of the Clusters' functioning. Some Clusters, such as those in Africa, are predominantly made up of Christians, but also have non-Christian members involved. If the ultimate goal of CHGN is to promote the development of holistic health and wellbeing around the globe, it must continue to seek innovative and appropriate ways to engage and partner with all types of organisations, regardless of their faith background.

During the forum, CHGN explored the formation of three additional new Clusters: Nepal, the Mekong region, and Bihar (India). Champions for these Clusters attended the forum and were able to glean important information to help them as they go forward. Drawing on their personal experiences and the evidence generated on Cluster formation, members from other Clusters and the CHGN hubs in the UK and Australia committed to helping these Clusters form and grow. ${ }^{10,12}$ This Cluster mentoring was seen by the forum participants as an important way to facilitate new Clusters to grow and effectively cooperate. This nurturing of new Clusters is also consistent with the CHGN vision and Clustering model.

To encourage sharing between different Clusters, it was recommended that communication and social media be more effectively utilised. Face-to-face interaction, however, was seen to be the most important medium to build collaboration between groups; therefore, annual regional forums and international forums every two to three years were recommended. Regular collaboration between Clusters from different countries appears to be an effective way of extending the work of Clusters from a local to a global scale.

\section{Conclusions and summary of learning}

\section{from the CHGN forum}

- Cluster formation at the community and primary care level provides a sustainable model for development and health-care provision, where local organisations formally link with one another for the purposes of collaboration, resource, and knowledge sharing.

- A common geographical and value system and a recognition that more can be achieved by working together, provides a unique platform for diverse organisations to come together in a collaborative relationship based on trust.

- Existing Clusters and the CHGN hubs in the UK and Australia need to play a facilitating role in the formation of new Clusters in other areas and countries.

- Improved inter-cluster communication is required and should be promoted through email groups, social media, and face-to-face meetings.

\section{References}

1. United Nations Development Programme. A new sustainable development agenda 2016 [Internet].

Available from:

http://www.undp.org/content/undp/en/home/sdgovervie w.html

2. Walley J, Tinker A, de Francisco A, Chopra M, Rudan I et al. Primary health care: Making Alma-Ata a reality. The Lancet. 2008;372(9642):1001-7. http://dx.doi.org/10.1016/S0140-6736(08)61409-9 
3. Grills, N. 'Believing' in HIV: The effect of faith on the response of Christian faith based organizations to HIV in India. [Doctor of Philosophy]. ND.

4. Community Health Global Network (CHGN). Collaborative networking for community health. 2014 [cited 2016 30/04/2016]. Available from: www.chgn.org

5. United Nations. MDG gap task force report. 2015 Contract No.: IBSN 78-92-1-101317-7.

6. United Nations Development Policy and Analysis Division (DESA). UN system task team on the post2015 UN development agenda 2012 [Internet].

Available from:

http://www.un.org/millenniumgoals/pdf/Think Pieces/8 health.pdf

7. Marmot M, Houweling T, Taylor S. Closing the gap in a generation: Health equity through action on the social determinants of health. The Lancet.

2008;372(9650):1661-9.

http://dx.doi.org/10.1016/S0140-6736(08)61690-6
8. Blas E, Gilson L, Kelly MP, Labonté R, Lapitan J and Muntaner $\mathrm{C}$ et al. Addressing social determinants of health inequities: What can the state and civil society do? The Lancet. 372(9650):1684-9. http://dx.doi.org/10.1016/S0140-6736(08)61693-1

9. The Lancet series on faith and global health. The Lancet. 2015;386(10005). http://dx.doi.org/10.1016/S0140-6736(15)60250-1

10. Safe M, Wainwright E, Lankaster T. Community health global network: "Clustering" together to increase the impact of community led health and development. CJGH. 2014;1(2):71-80. http://dx.doi.org/10.15566/cjgh.v1i2.9

11. 1 Corinthians. In: The holy Bible: English standard version. Illinois: Good News Publishers.

12. Grills N, Philip M, Porter G. Networking between community health programs: A team-work approach to improving health service provision. BMC Health Services Research. 2014;14(1). http://dx.doi.org/10.1186/1472-6963-14-297

Figure 1. Community Health Global Network International Forum May 2016

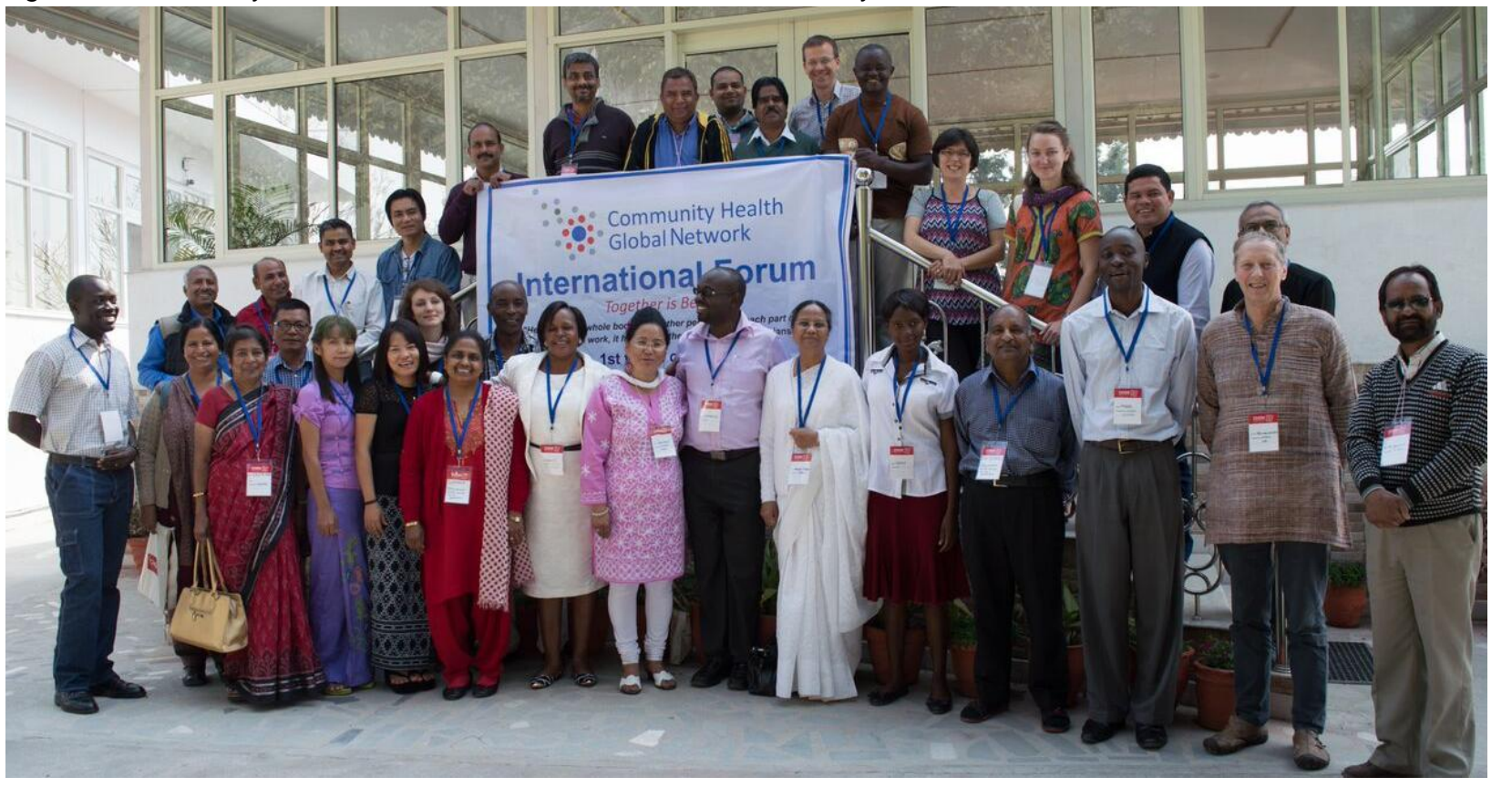

Competing Interests: None declared.

May 2016. Christian Journal for Global Health, 3(1): 89-94. 
Correspondence: For more information on CHGN please see www.chgn.org, or contact elizabeth.wainwright@chgn.org or grills@unimelb.edu.au

Cite this article as: Young, R, Hughes, N, Wainwright, E, Lankester, T, Grills, N. Community Health Global Network and sustainable development. Christian Journal for Global Health (May 2016), 3(1):89-94.

(C) Young, $R$ et al This is an open-access article distributed under the terms of the Creative Commons Attribution License, which permits unrestricted use, distribution, and reproduction in any medium, provided the original author and source are properly cited. To view a copy of the license, visit http://creativecommons.org/licenses/by/3.0/

$$
\text { www.cjgh.org }
$$

May 2016. Christian Journal for Global Health, 3(1): 89-94. 